\title{
Four Wing Flapping Micro Air Vehicles - Dragonflies or X-Wings?
}

\author{
Christopher T. Orlowski, Anouck R. Girard ${ }^{\dagger}$ and Wei Shyy $\ddagger$ \\ University of Michigan, Ann Arbor, MI, 48109, USA
}

\begin{abstract}
The aerodynamic feats of dragonflies are well documented. However, human beings have created flying vehicles that do not mimic nature, e.g. helicopters and quad-rotors. The paper presents initial investigations, via simulations, into the dynamics of a four wing, flapping wing micro-air vehicle. The paper attempts to answer the question of whether four wings in a traditional setup, akin to a dragonfly, is more, or less, beneficial than a ' $x$-wing' configuration. The micro-air vehicle is modeled as a system of five connected rigid bodies, a central body and four wings. The equations of motion are derived using D'Alembert's Principle for Multiple Rigid Bodies. Each wing is given three separate degrees of freedom relative to the central body. Open loop simulations are presented using the full nonlinear equations of motion, which include the inertial effects of the wings on the central body. Simulations show that an ' $x$-wing' configuration and a 'revised' dragonfly configuration may provide better, inherent stability than a biomimetic vehicle modeled after a dragonfly. Simulations of the two configurations show better pitch stability than a biomimetic simulation and the 'revised' dragonfly exhibits outstanding stability and lift characteristics.
\end{abstract}

\section{Nomenclature}

$\theta \quad$ pitch angle of the central body

$A_{w} \quad$ wing area

$\hat{c} \quad$ normalized chord

$C_{N} \quad$ normal force coefficient for calculation of aerodynamic forces

$C_{T} \quad$ tangential force coefficient for calculation of aerodynamic forces

$F_{\text {aero }} \quad$ aerodynamic forces expressed in the $B$ frame

$\mathbf{I}_{i}$ inertia tensor for the ith rigid body

$m_{i} \quad$ mass of the $i t h$ rigid body

$m_{\text {sys }} \quad$ mass of the system

$M_{\text {aero }} \quad$ aerodynamic moments expressed in the $B$ frame

$p, q, r$ angular velocity components of the central body in the body frame with respect to the inertial frame

$p_{R F}, q_{R F}, r_{R F}$

$p_{R H}, q_{R H}, r_{R H}$

$p_{L F}, q_{L F}, r_{L F}$

$p_{L H}, q_{L H}, r_{L H}$

$\hat{r}_{2}$

$\bar{r}_{k l}$ angular velocity components of the right forewing in the stroke plane frame

angular velocity components of the right hindwing in the stroke plane frame

angular velocity components of the left forewing in the stroke plane frame

angular velocity components of the left hindwing in the stroke plane frame

normalized wing center of pressure

$\mathbf{R}_{B}$ position vector of the wing joint from the origin of the body frame

$\mathbf{R}_{\beta_{k l}}$

$\mathbf{R}_{k l}$

$u, v, w$ rotation matrix from the inertial frame to the body frame rotation matrix from the body frame to the stroke plane frame rotation matrix from the stroke plane frame to the wing frame translational velocity components of the central body along in the body frame

${ }^{*} \mathrm{PhD}$ Candidate, Department of Aerospace Engineering, University of Michigan, Senior Member AIAA.

${ }^{\dagger}$ Assistant Professor, Department of Aerospace Engineering, University of Michigan, Associate Member AIAA.

‡Clarence L. "Kelly" Johnson Collegiate Professor and Chair, Department of Aerospace Engineering, University of Michigan, Fellow AIAA. 
with respect to the inertial frame

$\overline{\mathbf{v}}_{i} \quad$ velocity vector for the $i t h$ rigid body

$\alpha \quad$ angle of attack of the wing, rotation about the $y$-axis of the stroke plane frame

$\dot{\alpha} \quad$ time rate of change of the angle of attack

$\bar{\beta}_{i j}$

$\beta_{k l}$

$\bar{\gamma}_{i j}$

$\delta$

$\dot{\delta}$

$\zeta$

\section{Introduction}

The aerodynamic feats and performance of dragonflies are well known. Dragonflies have fascinated scientists for years ${ }^{1}$ and a large portion of research into dragonflies has focused on the wing kinematics and the associated force generation. Norberg produced a seminal work on dragonflies in 1975 and was one of the first biologists to determine the stroke kinematics, stroke plane angle, and proposed mechanisms for force generation of a dragonfly species. ${ }^{2}$ Wakeling and Ellington, in Refs. 1,3,4, analyzed the flight performance of the Ruddy Darter. Wang et. al measured the wing kinematics and flight trajectory of dragonflies during forward flight and turning maneuvers in Ref 5 .

Dragonflies present a desirable choice for implementation in a flight vehicle because the four wings provide additional lift over two wings and may provide more control authority over the vehicle. ${ }^{6}$ The paper presents presents a flight dynamics model of a four wing, flapping wing micro-air vehicle. The model does not assume that the mass and inertial effects of the wings are negligible. Simulations are presented that attempt to answer the question of whether a biomimetic or non-biomimetic design is more apt for vehicle implementation. The biomimetic model is based on a dragonfly, presented in Ref. 7. The non-biomimetic model is based of a design for wing actuation presented in Ref. 8, but uses the same body parameters as the biomimetic model for consistency. The design, referred to as the ' $\mathrm{x}$-wing' configuration, is not one that appears in nature. However, helicopters don't appear in nature, either. The flight dynamics model is derived using D'Alembert's Principle for Multiple Rigid Bodies, previously presented in Refs. 9,10. The flapping wing micro-air vehicle is modeled as a system of five rigid bodies, a central body and four wings. Each wing is given three degrees of freedom: flapping motion through the stroke plane, deviation from the stroke plane, and active pitching about the wing root.

The paper is presented in the following manner. Section II presents the dynamic model derivation. Section III presents the parameters used for simulation, including the body parameters and aerodynamic model. Section IV presents the simulations, including both the biomimetic and non-biomimetic models. Section $\mathrm{V}$ is the conclusion of the paper. 


\section{Dynamic Model Development}

The chosen method for the derivation of the equations of motion for a flapping wing micro-air vehicle is D'Alembert's Principle for Multiple Rigid Bodies. The statement of the method is

$$
\sum_{i=1}^{n}\left[m_{i}\left(\dot{\overline{\mathbf{v}}}_{i}+\ddot{\bar{\rho}}_{c i}\right) \cdot \bar{\gamma}_{\mathbf{i j}}+\left(\mathbf{I}_{\mathbf{i}} \cdot \dot{\bar{\omega}}_{\mathbf{i}}+\bar{\omega}_{\mathbf{i}} \times \mathbf{I}_{\mathbf{i}} \cdot \bar{\omega}_{\mathbf{i}}+m_{i} \bar{\rho}_{\mathbf{c i}} \times \dot{\overline{\mathbf{v}}}_{i}\right) \cdot \bar{\beta}_{\mathbf{i j}}\right]=Q_{j},
$$

where $i$ is the number of rigid bodies and $j$ is the number of generalized coordinates. A detailed description of the method can be found in Ref. 11 and Ref. 12. The method is a combination of Eulerian and Lagrangian techniques. ${ }^{13}$ The model is composed of five rigid bodies: the central body $(i=1)$, the right forewing $(i=2)$, the right hindwing $(i=3)$, the left forewing $(i=4)$, and the left hindwing $(i=5)$. In the case of the $\mathrm{x}$-wing configuration, the top wings will be the forewings and the bottom wings will be the hindwings. A model of the two cases, for representation purposes only, is shown in Figure 1. The method of derivation was previously used to develop the equations of motion for a two-wing flapping wing micro-air vehicle ${ }^{9}$ and a flapping wing micro-air vehicle with a tail and a control mass. ${ }^{10}$ Simulations will investigate four main kinematic configurations, two for the dragonfly configuration and two for the $\mathrm{x}$-wing configuration. The dragonfly configuration will be investigated with biomimetic wing kinematics. The dragonfly configuration will also be presented with 'revised' wing kinematics, where the wings beat in a horizontal stroke plane and the motion of the forewing is equal and opposite to the motion of the hindwings. The $\mathrm{x}$-wing configuration will be presented with the wing kinematics constrained to a horizontal stroke plane and a vertical stroke plane, where the stroke plane is relative to the longitudinal avic of tho nontrol hodvr

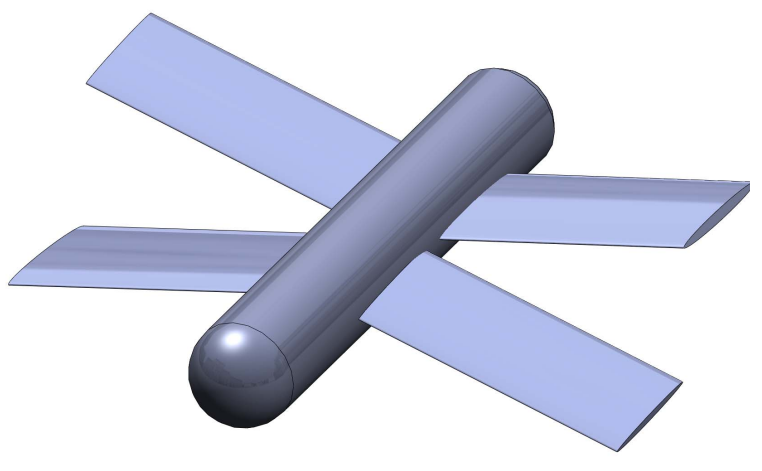

(a) Dragonfly

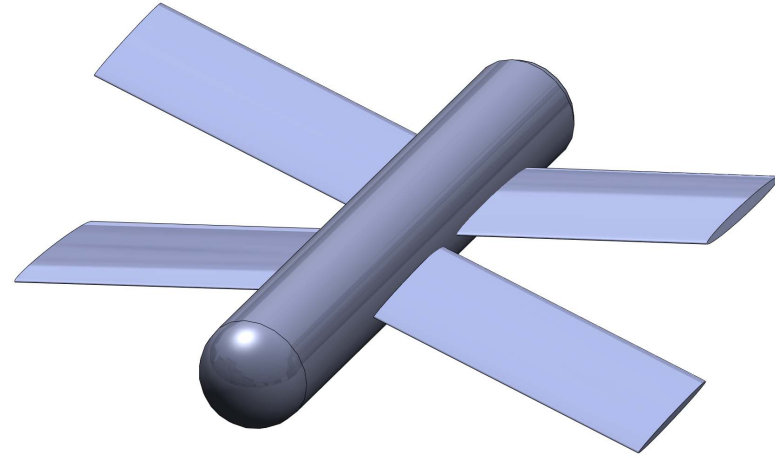

(b) X-Wing

Figure 1. Model Representations

\section{A. Assumptions}

The flapping wing micro-air vehicle is operating in a flat Earth environment with constant gravity and zero wind. We assume no aerodynamic interactions between the wings, specifically the forewings and hindwings, and no aerodynamic interactions between the wings and the central body. The wings are attached at joints that provide each wing with three degrees of freedom: fore and aft motion within the stroke plane, active pitching of the wing about the wing root, and deviation from the stroke plane. ${ }^{14}$ The central body and all four wings are each considered to be rigid bodies with constant mass properties.

\section{B. Reference Frames}

A body-fixed frame, henceforth referred to as the $B$ frame, is fixed to the central body. The origin of the $B$ frame is the center of mass of the central body. The frame is oriented with the $x$-axis along the longitudinal axis of the central body, the $y$-axis perpendicular to the $x$-axis and positive out of the right side of the vehicle, and the $z$-axis is perpendicular to the $x-y$ plane and positive downward. The units vectors for the $B$ frame are $\hat{b}_{x}, \hat{b}_{y}$, and $\hat{b}_{z}$. A diagram of the $B$ frame is depicted in Figure 2. The orientation of the body with respect to an inertial frame is obtained using the standard 3-2-1 Euler angles for aircraft, where $\psi$ denotes 
the yaw angle, $\theta$ denotes the pitch angle, and $\phi$ denotes the roll angle. The rotation matrix describing the orientation of the $B$ frame with respect to an inertial frame is denoted by $\mathbf{R}_{B}$, the full details of 3-2-1 Euler angles for aircraft can be found in Refs. 13 and 15.

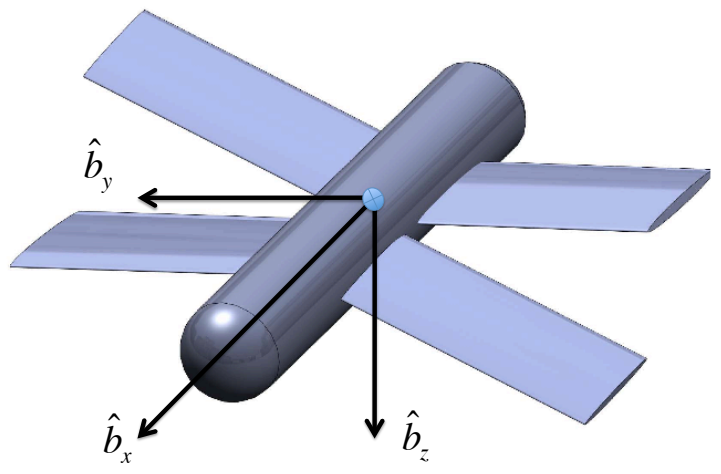

Figure 2. $B$ Frame

In addition to the $B$ frame, stroke plane frames are fixed at each of the wing joints. The origin of the four stroke plane frames, one for each of the wings, is the wing joint. The $y$-axis of the stroke plane frames is parallel to the $\hat{b}_{y}$ axis of the body frame. The $x$-axis and the $z$-axis of the stroke plane frame are rotated, with respect to the $B$ frame, by the stroke plane angle $\beta$. The relation between the stroke plane and the $B$ frame is depicted in Figure 3, along with the angle of attack of the wing relative to the stroke plane. The rotation matrix describing the orientation between the $B$ frame and the stroke plane frame is

$$
\mathbf{R}_{\beta}=\left[\begin{array}{ccc}
\cos \beta & 0 & -\sin \beta \\
0 & 1 & 0 \\
\sin \beta & 0 & \cos \beta
\end{array}\right],
$$

where there are individual rotation matrices for each of the four stroke planes with angles $\beta_{R F}, \beta_{R H}, \beta_{L F}$, and $\beta_{L H}$. If the stroke plane angle is identically zero, then the stroke plane frame is parallel to the $B$ frame. In addition to the stroke plane frame, fixed-frames are attached to each of the four wings. The origins of

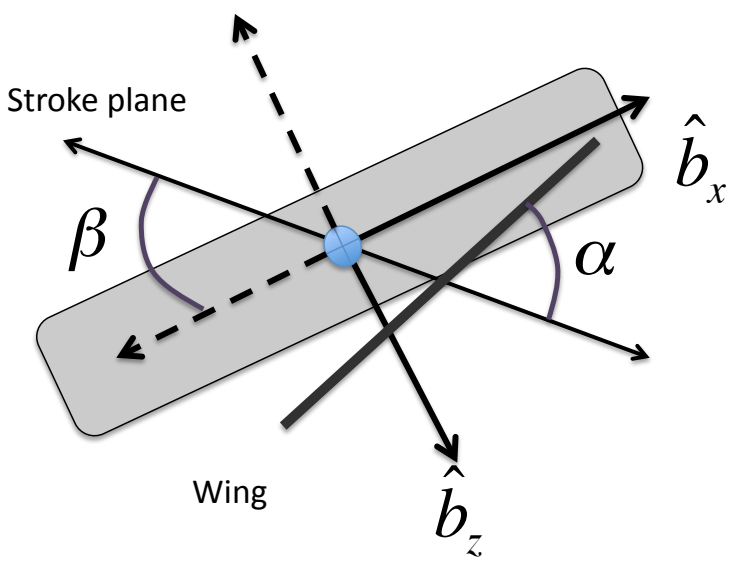

Figure 3. Stroke Plane, $\beta$, and Angle of Attack, $\alpha$ 
each of the wing frames is the wing root, which is coincident with the wing joint. The initial orientation of the wing frames is parallel to the stroke plane frames. However, the wing frames move with the wings as they flap and allow for the determination of the orientation of the wing with respect to the stroke plane frame, and, by extension, the $B$ frame. The orientation of the wing frames is described by the deviation angle $\delta$, the angle of attack (or pitch angle) $\alpha$, and the flapping angle $\zeta$. Positive rotations for each of the angles follow the right hand rule. A depiction of the deviation and flapping angles appears in Figure 4 . The

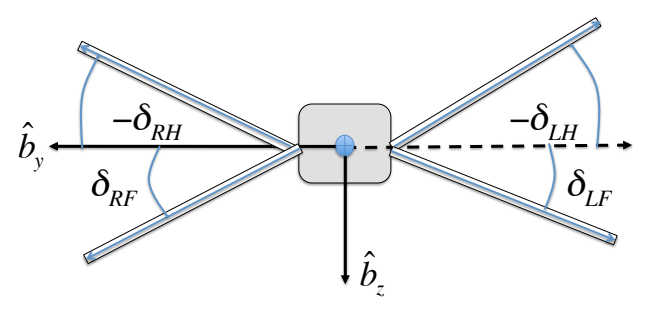

(a) Deviation ( $\delta$ ) Angles

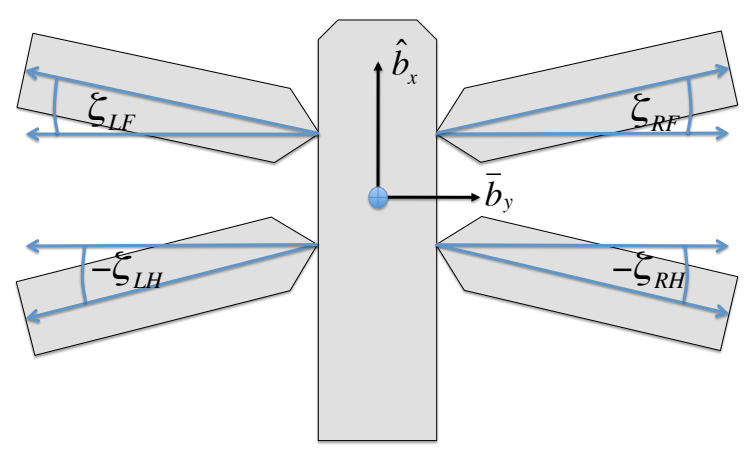

(b) Flapping ( $\zeta)$ Angles

Figure 4. Deviation and Flapping Angles

rotation matrices for the wings on the right side of the vehicle (positive $\hat{b}_{y}$ ) are the following

$$
\mathbf{R}_{\delta_{R}}=\left[\begin{array}{ccc}
1 & 0 & 0 \\
0 & \cos \delta_{R} & -\sin \delta_{R} \\
0 & \sin \delta_{R} & \cos \delta_{R}
\end{array}\right], \quad \mathbf{R}_{\alpha_{R}}=\left[\begin{array}{ccc}
\cos \alpha_{R} & 0 & -\sin \alpha_{R} \\
0 & 1 & 0 \\
\sin \alpha_{R} & 0 & \cos \alpha_{R}
\end{array}\right], \quad \text { and } \quad \mathbf{R}_{\zeta_{R}}=\left[\begin{array}{ccc}
\cos \zeta_{R} & \sin \zeta_{R} & 0 \\
-\sin \zeta_{R} & \cos \zeta_{R} & 0 \\
0 & 0 & 1
\end{array}\right]
$$

The orientation of the wing frame with respect to the stroke plane frame is obtained by 3-1-2 Euler angles in the following manner

$$
\mathbf{R}_{R}=\mathbf{R}_{\alpha_{R}} \mathbf{R}_{\delta_{R}} \mathbf{R}_{\zeta_{R}} .
$$

The rotation matrices for the right forewing and the right hindwing are identical in form, except for the substitution of the proper angle, e.g. $\delta_{R}=\delta_{R F}=\delta_{R H}$. The left wings (negative $\hat{b}_{y}$ ) are on the opposite side of the vehicle and as a result, the rotation matrices differ from those on the right hand side. The rotation matrices for the left wing are

$$
\mathbf{R}_{\delta_{L}}=\left[\begin{array}{ccc}
1 & 0 & 0 \\
0 & \cos \delta_{L} & \sin \delta_{L} \\
0 & -\sin \delta_{L} & \cos \delta_{L}
\end{array}\right], \quad \mathbf{R}_{\alpha_{L}}=\left[\begin{array}{ccc}
\cos \alpha_{L} & 0 & -\sin \alpha_{L} \\
0 & 1 & 0 \\
\sin \alpha_{L} & 0 & \cos \alpha_{L}
\end{array}\right], \quad \text { and } \quad \mathbf{R}_{\zeta_{L}}=\left[\begin{array}{ccc}
\cos \zeta_{L} & -\sin \zeta_{L} & 0 \\
\sin \zeta_{L} & \cos \zeta_{L} & 0 \\
0 & 0 & 1
\end{array}\right] \text {. }
$$

The rotation matrices for the left wings are combined in the same manner as for the right wing, as shown in Equation 4. The opposite negative signs in the rotation matrices for the $\delta$ and $\zeta$ angles ensure proper cancellation of forces and moments in the $B$ frame, when the motion of the wings is symmetric with respect to the central body.

\section{Generalized Coordinates}

The number of generalized coordinates for the system is equal to the total number of degrees of freedom of the system. The system truly only has six degrees of freedom: the three translational and rotational degrees of freedom of the central body. The degrees of freedom for each of the wings are holonomically constrained to the central body. However, we can consider the wing motions as separate degrees of freedom, while acknowledging the fact that the system truly has six degrees of freedom. ${ }^{11}$ The coordinates that describe 
the six degrees of freedom of the central body are its inertial position $(X, Y, Z)$ and orientation with respect to the inertial frame $(\psi, \theta, \phi)$. The additional coordinates in the system are the wing angles for each of the four wings $(\delta, \alpha, \zeta)$. Sum total, the eighteen generalized coordinates, $\mathbf{q}_{j}$, for the system are

$$
\mathbf{q}_{j}=\left[\begin{array}{ccccccccc}
X & Y & Z & \psi & \theta & \phi & \delta_{R F} & \alpha_{R F} & \zeta_{R F} \\
\delta_{R H} & \alpha_{R H} & \zeta_{R H} & \delta_{L F} & \alpha_{L F} & \zeta_{L F} & \delta_{L H} & \alpha_{L H} & \zeta_{L H}
\end{array}\right] .
$$

The $j$ generalized coordinates are numbered from left to right and top to bottom in Equation 6 , e.g. $X(j=1)$ and $\delta_{R H}(j=10)$. The associated quasi-velocities, $\mathbf{u}_{j}$ are associated with degrees of freedom of the central body and each of the four wings. The quasi-velocities for the central body are the translational velocities $(u, v, w)$ and the angular velocities $(p, q, r)$, both expressed in the $B$ frame with respect to the inertial frame. The quasi-velocities for the wings are the angular velocities of the wings with respect to the stroke plane frames (for example, $p_{R H}, q_{R H}$, and $r_{R H}$ ). The eighteen quasi-velocities are

$$
\mathbf{u}_{j}=\left[\begin{array}{ccccccccc}
u & v & w & p & q & r & p_{R F} & q_{R F} & r_{R F} \\
p_{R H} & q_{R H} & r_{R H} & p_{L F} & q_{L F} & r_{L F} & p_{L H} & q_{L H} & r_{L H}
\end{array}\right] .
$$

\section{Reference Vectors}

The reference vectors are denoted by $\bar{\rho}_{c i}$ in Equation 1 . The reference vectors describe the position of the center of mass of the ith rigid body with respect to a reference point for the entire system. The reference point for the system is chosen to be the origin of the $B$ frame, coincident with center of mass of the central body. As a result, the reference vector for the central body, $\bar{\rho}_{c 1}$, is identically zero. The wings are modeled as thin, flat plates, with the assumption that the center of mass of the wing lies along the $y$-axis of the wing frame. The center of mass of each of the wings is located at a distance, $\rho_{i}$ along the $y$-axis of each of the wings. In the stroke plane, the center of mass of each of the wings is tracked by a combination of the angles $\delta$ and $\zeta$. For a horizontal stroke plane, $\beta \equiv 0$, the reference vector for the right forewing, expressed in the $B$ frame, has the form

$$
\bar{\rho}_{c 2}=\rho_{2}\left[\begin{array}{c}
\cos \delta_{R F} \sin \zeta_{R F} \\
\cos \delta_{R F} \cos \delta_{R F} \\
\sin \delta_{R F}
\end{array}\right],
$$

where $R H$ and $\rho_{3}$ are substituted for $R F$ and $\rho_{2}$ in Equation 8 for the right hindwing. The left wings are on the opposite side of the aircraft and the reference vectors differ in the $\hat{b}_{y}$ component. With the stroke plane angle identically zero, the reference vector for the left forewing, expressed in the $B$ frame, is

$$
\bar{\rho}_{c 4}=\rho_{4}\left[\begin{array}{c}
\cos \delta_{L F} \sin \zeta_{L F} \\
-\cos \delta_{L F} \cos \delta_{L F} \\
\sin \delta_{L F}
\end{array}\right],
$$

where $L H$ and $\rho_{5}$ are substituted for $L F$ and $\rho_{4}$ in Equation 9 for the left hindwing reference vector $\bar{\rho}_{c 5}$. When the stroke plane angle is not identically zero, the reference vectors are expressed in the $B$ frame through a rotation from the stroke plane. For example, the reference vector for the right hindwing is the following

$$
\bar{\rho}_{c 3}=\rho_{3} \mathbf{R}_{\beta_{R H}}^{T} \rho_{c 3, s p} .
$$

The acceleration of each of the reference vectors, denoted by $\ddot{\bar{\rho}}_{c i}$ in Equation 1, is obtained by thorough use of the Transport Theorem and the fact that the stroke plane angle is assumed to remain constant.

\section{E. Velocities}

The translational and angular velocities of each of the five rigid bodies is necessary to complete the equations of motion. The velocity of the central body, expressed in the $B$ frame, is

$$
\overline{\mathbf{v}}_{1}=u \hat{b}_{x}+v \hat{b}_{y}+w \hat{b}_{z} .
$$

The angular velocity of the central body is

$$
\bar{\omega}_{1}=p \hat{b}_{x}+q \hat{b}_{y}+r \hat{b}_{z} .
$$


In order to simplify the dynamics, and eliminate the requirement of tracking the absolute velocity and accelerations of each of the wings, the reference velocity for each of the wings is chosen to be the velocity of the respective wing joint. In order to express the velocity of the wing joint in the $B$ frame, we define a vector from the origin of the $B$ frame to each of the wing joints. The four vectors, $\bar{r}_{R F}, \bar{r}_{R H}, \bar{r}_{L F}, \bar{r}_{L H}$, are fixed in the $B$ frame, and since the central body is assumed to be rigid, the vectors are constant. The orientation of the vectors to the wing joints and the reference vectors for each of the rigid bodies detailed in Section $\mathrm{D}$ are shown in Figure 5.

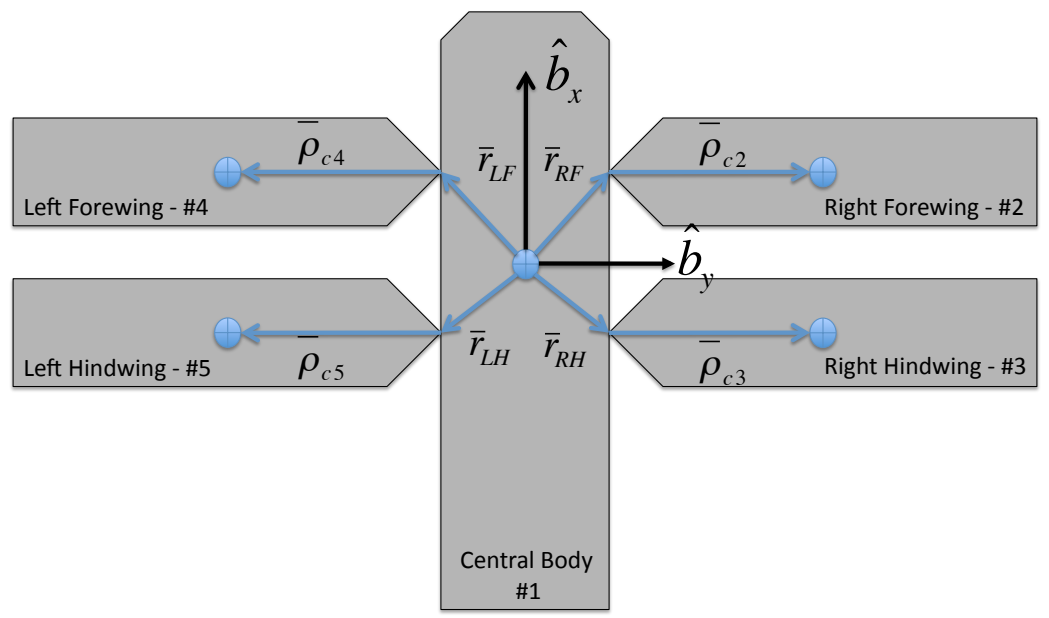

Figure 5. Reference Vectors

Each of the vectors to the wing joints may, or may not, have components in all three directions of the $B$ frame, depending on the configuration of the system. The translational velocity of the wing joints will be a combination of the translational and angular velocities of the central body and the wing joint vector. For example, the translational velocity for the left hindwing joint, is

$$
\overline{\mathbf{v}}_{5}=\overline{\mathbf{v}}_{1}+\bar{\omega}_{1} \times \bar{r}_{L H} .
$$

The angular velocities of the wings are a vector sum of the angular velocity of the central body and the angular velocity of the respective wing. We define a positive angular velocity to be consistent with the right hand rule. As a result, and consistent with the development of the rotation matrices for each of the wings, the velocities $\dot{\delta}$ and $\dot{\zeta}$ will be opposite in sign for the right and left wings. The angular velocity of the wings, with respect to the respective stroke plane frame, is obtained from the 3-1-2 Euler angle relationship between the orientation of the wings and the stroke plane frame. The angular velocity of the right forewing, in the stroke plane frame, is obtained according to

$$
\bar{\omega}_{2, s p}=\left[\begin{array}{c}
p_{R F} \\
q_{R F} \\
r_{R F}
\end{array}\right]=\mathbf{R}_{R F}\left[\begin{array}{c}
0 \\
0 \\
-\dot{\zeta}_{R F}
\end{array}\right]+\mathbf{R}_{\alpha_{R F}} \mathbf{R}_{\delta_{R F}}\left[\begin{array}{c}
\dot{\delta}_{R F} \\
0 \\
0
\end{array}\right]+\mathbf{R}_{\alpha_{R F}}\left[\begin{array}{c}
0 \\
\dot{\alpha}_{R F} \\
0
\end{array}\right] .
$$

The angular velocity of the right hindwing, $\bar{\omega}_{3, s p}$, is obtained in the same manner, with the substitution of $R H$ for $R F$. The angular velocity of the left forewing, with respect to the stroke plane frame is calculated from

$$
\bar{\omega}_{4, s p}=\left[\begin{array}{c}
p_{L F} \\
q_{L F} \\
r_{L F}
\end{array}\right]=\mathbf{R}_{L F}\left[\begin{array}{c}
0 \\
0 \\
\dot{\zeta}_{L F}
\end{array}\right]+\mathbf{R}_{\alpha_{L F}} \mathbf{R}_{\delta_{L F}}\left[\begin{array}{c}
-\dot{\delta}_{L F} \\
0 \\
0
\end{array}\right]+\mathbf{R}_{\alpha_{L F}}\left[\begin{array}{c}
0 \\
\dot{\alpha}_{L F} \\
0
\end{array}\right] .
$$


The angular velocity of the left hindwing, $\bar{\omega}_{5, s p}$, is obtained using the same equation, but substituting for the left hindwing. The description of the angular velocities is consistent with angular velocity development in Ref. 16. When the stroke plane is not identically zero, the total angular velocities of the wings, when expressed in the $B$ frame, will differ. For a non-zero stroke plane angle $\beta_{R H}$, the angular velocity of the right hindwing is

$$
\bar{\omega}_{3}=\mathbf{R}_{\beta_{R H}}{ }^{\mathrm{T}} \bar{\omega}_{3, s p}
$$

The angular velocity for the left hindwing, for a non-zero $\beta_{L H}$, is

$$
\bar{\omega}_{5}=\mathbf{R}_{\beta_{L H}}{ }^{\mathrm{T}} \bar{\omega}_{5, s p}
$$

\section{F. Velocity and Angular Velocity Coefficients}

To complete the equations of motion, the velocity and angular velocity coefficients need to be calculated. The coefficients are calculated according to

$$
\bar{\gamma}_{i j}=\frac{\partial \overline{\mathbf{v}}_{i}}{\partial \mathbf{u}_{j}} \quad \text { and } \quad \bar{\beta}_{i j}=\frac{\partial \bar{\omega}_{i}}{\partial \mathbf{u}_{j}},
$$

where $\bar{\gamma}_{i j}$ is the velocity coefficient for the $i t h$ body with respect to the $j$ th quasi-velocity and $\bar{\beta}_{i j}$ is the angular velocity coefficient for the $i t h$ rigid body with respect to the $j t h$ quasi-velocity. Combining the five rigid bodies with the eighteen generalized coordinates results in the calculation of ninety velocity coefficients and ninety angular velocity coefficients. The velocity and angular velocity coefficients are combined with the other components of the derivation according to Equation 1. Each velocity and angular velocity coefficient is a vector. For example, the velocity and angular velocity coefficients for $i=1$ and $j=1$ are

$$
\bar{\gamma}_{11}=\frac{\partial \overline{\mathbf{v}}_{1}}{\partial u}=\hat{b}_{x} \quad \text { and } \quad \bar{\beta}_{11}=\frac{\partial \bar{\omega}_{1}}{\partial u}=0
$$

The result is the contribution of the central body to the first equation of motion, as shown in Equation 20

$$
\begin{gathered}
m_{1}\left(\dot{\overline{\mathbf{v}}}_{1}\right) \cdot \bar{\gamma}_{11}+\left(\mathbf{I}_{1} \dot{\bar{\omega}}_{1}+\bar{\omega}_{1} \times \mathbf{I}_{1} \bar{\omega}_{1}\right) \cdot \bar{\beta}_{11}=Q_{1} \\
m_{1}\left(\dot{\overline{\mathbf{v}}}_{1}\right) \cdot \bar{b}_{x}+\left(\mathbf{I}_{1} \dot{\overline{\bar{\omega}}}_{1}+\bar{\omega}_{1} \times \mathbf{I}_{1} \bar{\omega}_{1}\right) \cdot 0=Q_{1} \\
m_{1}(\dot{u}+q w-r v)=Q_{1}
\end{gathered}
$$

In total, the derivation will result in eighteen, second-order equations of motion.

\section{G. Forces and Moments}

The aerodynamic forces and moments acting on the central body are a combination of the forces and moments produced by the motion of each wing, expressed in the $B$ frame. The aerodynamic forces are denoted by

$$
\bar{F}_{\text {aero }}=F_{x} \hat{b}_{x}+F_{y} \hat{b}_{y}+F_{z} \hat{b}_{z}
$$

The aerodynamic moments are denoted as

$$
\bar{M}_{\text {aero }}=L \hat{b}_{x}+M \hat{b}_{y}+N \hat{b}_{z} .
$$

The forces and moments for each coordinate, $Q_{j}$, are calculated according to the Principle of Virtual Work. ${ }^{11}$ The forces affecting the translation of the central body are

$$
\left[\begin{array}{l}
Q_{1} \\
Q_{2} \\
Q_{3}
\end{array}\right]=\left[\begin{array}{l}
F_{x} \\
F_{y} \\
F_{z}
\end{array}\right]+\left(m_{s y s}\right) \mathbf{R}_{B}\left[\begin{array}{l}
0 \\
0 \\
g
\end{array}\right]
$$

The moments affecting the rotation of the central body are

$$
\left[\begin{array}{l}
Q_{4} \\
Q_{5} \\
Q_{6}
\end{array}\right]=\bar{M}_{a e r o}+\bar{M}_{g, R F}+\bar{M}_{g, R H}+\bar{M}_{g, L F}+\bar{M}_{g, L H}
$$


where the moments due to gravity of the wings have the following form

$$
\bar{M}_{g, R F}=\left(\bar{r}_{R F}+\bar{\rho}_{c 2}\right) \times\left(m_{2}\right) \mathbf{R}_{B}\left[\begin{array}{l}
0 \\
0 \\
g
\end{array}\right] .
$$

The generalized moments for the equations governing the motions of the wing are $Q_{j}$ for $j=7 \ldots 18$, where each control moment results in the proper angular acceleration of each wing.

\section{Simulation Model}

\section{A. Body Parameters}

The central body is modeled after the Sympetrum sanguineum, the Ruddy Darter dragonfly, studied by Wakeling and Ellington in Refs. 1,3,4,7. The specific insect chosen is specimen SSan1. The mass of the central body is set at $127.4 \mathrm{mg}$. The body is modeled as a solid cylinder with a length of $34 \mathrm{~mm}$, a constant radius of $5 \mathrm{~mm}$, and a constant mass distribution. The center of mass of the central body, for simulation purposes, is assumed to be at $17 \mathrm{~mm}$ from the nose of the aircraft, along the $\hat{b}_{x}$ axis.

Each of the wings is modeled as a thin, flat plate with negligible thickness. The forewings mass is set at $1.57 \mathrm{mg}$ with a span of $28.62 \mathrm{~mm}$ each. The constant chord is selected based on the wing area in Ref. 7. For the forewings, the wing area is $341.66 \mathrm{~mm}^{2}$, resulting in a chord of $11.94 \mathrm{~mm}$. The hindwings' mass is set at $1.72 \mathrm{mg}$, with a span of $27.63 \mathrm{~mm}$ and a constant chord of $16.06 \mathrm{~mm}$, from a wing area of $443.77 \mathrm{~mm}^{2}$.

\section{B. Aerodynamic Model}

The aerodynamic model utilized for the simulations is developed and presented in Refs. 17-19. The normal and tangential components of the forces on the wing are calculated according to a quasi-steady model approach. The normal and tangential force coefficients are calculated according to

$$
\begin{gathered}
C_{N}(\alpha)=-\operatorname{sign}(\dot{\zeta}) 3.4 \sin \alpha \\
C_{T}(\alpha)=\left\{\begin{array}{cc}
-\operatorname{sign}(\dot{\zeta}) 0.4 \cos ^{2}(2 \alpha) & 0 \leq \alpha \leq 45^{\circ} \\
0 & \text { otherwise }
\end{array}\right.
\end{gathered}
$$

where $\alpha$ is the angle of attack of the wing relative to the stroke plane, see Figure 3. The use of the sign function ensures proper orientation of the normal and tangential forces, relative to the stroke plane. The normal and tangential forces on the wing, due to the translation of the wing, are

$$
F_{t r, N}(t)=\frac{1}{2} \rho A_{w} C_{N}(\alpha(t)) U_{c p}^{2}(t) \quad \text { and } \quad F_{t r, T}(t)=\frac{1}{2} \rho A_{w} C_{T}(\alpha(t)) U_{c p}^{2}(t) .
$$

The normal force on the wing, due to the rotation of the wing, is

$$
F_{r o t, N}(t)=\frac{1}{2} \rho A_{W} C_{r o t} c_{\text {max }} \dot{\alpha}(t) U_{c p}(t) .
$$

In Equations 27 and 28, $A_{w}$ is the wing area, $R$ is the wing length, and $U_{c p}$ is the velocity of the center of pressure of the wing. The additional parameters, which require further calculation based on wing geometry, are the normalized center of pressure, $\hat{r}_{2}$, and the normalized rotational chord, $\hat{c}$. The normalized center of pressure is calculated according to Equation 29 and the normalized rotational chord is calculated according to Equation 30.

$$
\begin{gathered}
\hat{r}_{2}=\sqrt{\frac{\int_{0}^{R} c(r) r^{2} d r}{R^{2} A_{w}}} \\
\hat{c}=\frac{\int_{0}^{R} c^{2}(r) r d r}{\hat{r}_{2} R A_{w} c_{\max }}
\end{gathered}
$$


The normal and tangential forces are calculated in the wing frame, denoted by $\bar{F}_{\text {wing }}$. The forces are transformed into the body frame in the following manner, using the right forewing as an example:

$$
\bar{F}_{\text {aero }, R F}=\mathbf{R}_{\beta_{R F}}{ }^{\mathrm{T}} \mathbf{R}_{R F}{ }^{\mathrm{T}} \bar{F}_{\text {wing }, R F} .
$$

The aerodynamic moments are calculated in the $B$ frame according to Equation 32, using the left hindwing as as an example,

$$
\bar{M}_{a e r o, L H}=\left(\bar{r}_{L H}+\bar{\rho}_{a c, 5}\right) \times \bar{F}_{a e r o, L H},
$$

where $\bar{\rho}_{a c, 5}$ is the position of the aerodynamic center of pressure of the wing in the $B$ frame. The aerodynamic center of pressure is assumed to have coordinates of $\left[\begin{array}{llll}\frac{c}{4} & \hat{r}_{2, R} b_{R} & 0\end{array}\right]^{T}$ in the wing frames for the right wings and $\left[\begin{array}{lll}\frac{c}{4} & -\hat{r}_{2, L} b_{L} & 0\end{array}\right]^{T}$ in the wing frame for the left wings, where $c$ is the chord of the respective wing and $b$ is the wingspan. In the $B$ frame, the aerodynamic center of pressure is calculated according to Equation 33 , which is demonstrated for the left hindwing:

$$
\bar{\rho}_{a c, 4}=\mathbf{R}_{\beta_{L H}}{ }^{\mathrm{T}} \mathbf{R}_{L H}^{\mathrm{T}}\left[\begin{array}{c}
\frac{c}{4} \\
-\hat{r}_{2, L H} b_{L H} \\
0
\end{array}\right] .
$$

The position of the aerodynamic center is valid when the angle of attack of the wing is positive, relative to the stroke plane. However, based on the Euler angle development for the wings, when the angle of attack is negative, the position of the center of pressure is at an $x$ coordinate of $-\frac{c}{4}$ in the wing frame. ${ }^{20-23}$ The difference in the position of the aerodynamic center is accounted for by an IF statement in the simulation code. The IF statement appears in the section of the code calculating the resultant aerodynamic moments. The aerodynamic forces are calculated, in the $B$ frame, according to Equation 31. The total aerodynamic forces acting on the central body, due to the motion of the wings, are

$$
\bar{F}_{\text {aero }}=\bar{F}_{\text {aero }, R F}+\bar{F}_{\text {aero }, R H}+\bar{F}_{\text {aero }, L F}+\bar{F}_{\text {aero }, L H}
$$

and the total aerodynamic moments are

$$
\bar{M}_{a e r o}=\bar{M}_{a e r o, R F}+\bar{M}_{a e r o, R H}+\bar{M}_{a e r o, L F}+\bar{M}_{a e r o, L H} .
$$

\section{Simulation Notes}

In order to transform the eighteen, second-order equations derived using Equation 1, the equations are rewritten in the following form

$$
\mathbf{A} \dot{\bar{u}}_{j}=\mathbf{B}
$$

where $\dot{\bar{u}}_{j}$ is a vector of the time derivatives of the quasi-velocities. Equation 36 is numerically solved using the ODE15s suite in MATLAB $囚$. The total model has a state space of thirty-six states. The simulations require an additional eighteen first-order differential equations. Three of the equations transform the translational velocities to an inertial frame and allow for integration of the inertial position. ${ }^{15}$ Three of the equations transform the angular velocities into Euler angle rates and allow for the integration of the Euler angles. The final twelve equations transform the angular velocities of the wings to the time derivatives of the wing angles, according to the proper Euler angle relationships.

\section{Results}

\section{A. Wing Kinematics and Dynamic Response of the System}

The following four subsections present simulation results for the model derived in Section II. The wing kinematics for each of the simulations are presented, detailing the stroke and angle of attack of the wings as functions of time. The kinematics presented for the traditional dragonfly in Section B are intended to be biomimetic and are not altered to investigate better performance. The kinematics presented for all other simulations were tested and changed to determine if certain configurations gave better, or worse, performance. As would be expected, if the wings are flapping faster, more lift and drag are generated. If the wings don't flap fast enough, or the stroke is to short (resulting in a slower $\dot{\zeta}$ for a given frequency), the models don't produce enough lift and will quickly fall from the sky. 
For the most part, the angle of attack for the wings is set to be positive, relative to the stroke plane frame, when the motion of the wing is forward (downstroke). The angle of attack is negative in the wing frame on the upstroke, but still results in a 'positive' angle of attack since the wing's motion is now reversed. If the wings are not phased properly, the aerodynamic moments can cause yaw, roll, and pitch instabilities. Specifically, the aerodynamic pitching moment is highly sensitive to the phase difference between the wing's stroke motion and angle attack. If the wingstrokes are symmetric about the longitudinal axis of the body, the cycle-averaged pitching moment should equal zero. ${ }^{20-23}$ However, it does not mean the aerodynamic moment won't have a drastic effect on the orientation and stability of the central body in a highly coupled, nonlinear system.

An example of the sensitivity of the aerodynamic moment due to the phase difference between the angle of attack and the stroke is shown in Figure 6 . Figure 6 shows the qualitative and quantitative difference for a

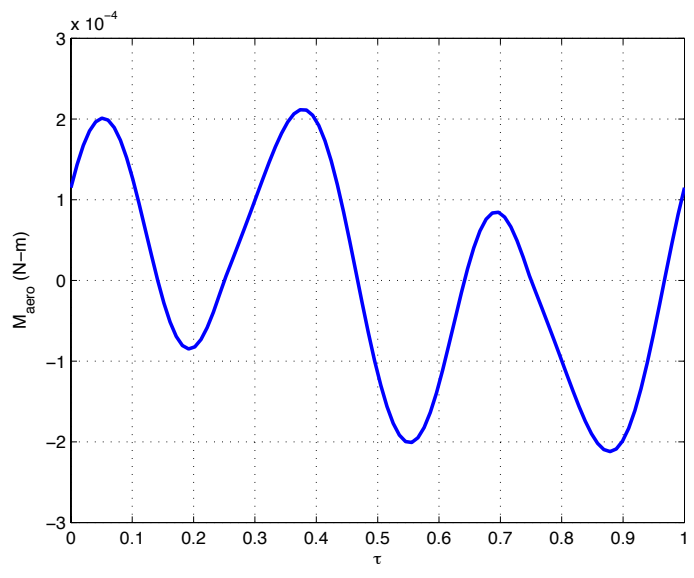

(a) $\alpha=45 \sin \left(2 \pi f t+\frac{\pi}{2}\right)$

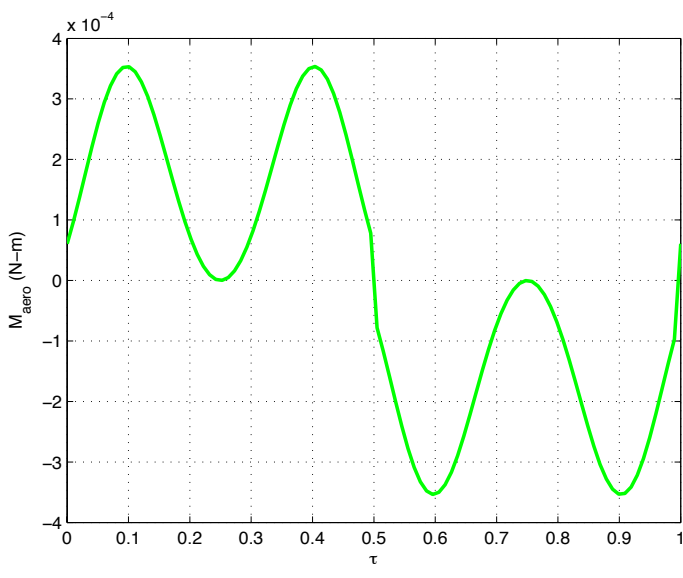

(b) $\alpha=45 \sin (2 \pi f t)$

Figure 6. $M_{\text {aero }}$ for $\zeta=\mathbf{6 0} \sin (2 \pi f t)$

phase shift in the angle of attack, without the same phase change in the stroke kinematics. The aerodynamic pitching moment with a phase shift of $\frac{\pi}{2}$ is cycle-averaged to zero. A phase shift of $\frac{\pi}{2}$ equates to a maximum angle of attack at mid-stroke and an angle of attack of zero at the end of the half-stroke. The cycle-average of the moment with a phase shift of zero is on the order of $10^{-5} N-m$. A phase shift of zero equates to a maximum angle of attack at the end of the half-strokes and an angle of attack of zero at mid-stroke. Dynamically, a system response for the first pitching moment has a slowly changing pitch angle, as the initial increase positive aerodynamic moment is compensated for by the gravity moment of the wings. The system with a phase shift equal to zero performs poorly and the non-zero pitching moment causes a drastic increase in the pitch angle in as little as five flapping cycles. Although the kinematics of the wings only come into play in the aerodynamic force and generation moment in the system, small changes can have a drastic effect on the performance of the system. The simulations presented are a 'best effort' with wingstrokes designed to aid the performance of the aircraft where possible.

\section{B. Normal Dragonfly}

The wings are arranged according to a dragonfly with the wingstrokes modeled, to a certain degree, after biological flyers. The forewings beat at a frequency of $38.7 \mathrm{~Hz}$ with a stroke plane angle, $\beta$, of 19.3 degrees. The hindwings beat at a frequency of $39.2 \mathrm{~Hz}$ with a stroke plane angle of 21.1 degrees. The stroke parameters are found in Ref. 3. Depending on the flight condition, the forewings and hindwings of a dragonfly will be in-phase or 90-180 degrees out of a phase. Furthermore, as found in Ref. 2, the wingbeat is not necessarily symmetrical about the longitudinal axis of the central body. We have chosen the hindwings to lead the forewings by 180 degrees. The lagging angles for the fore- and hindwings are the following

$$
\zeta_{F}(t)=5+30 \sin \left(2 \pi f_{F} t\right) \text { and } \zeta_{H}(t)=15+30 \sin \left(2 \pi f_{H} t+\pi\right),
$$

where $f_{F}$ is the frequency of the forewings, $f_{H}$ is the frequency of the hindwings, and the angle is given in degrees. The maximum amplitude of the wings is 30 degrees, giving a total stroke amplitude of 60 degrees. 
Traditionally, a dragonfly maintains a constant angle of attack during the downstroke, which is nearly perpendicular to the velocity of the wing, while on the upstroke the chord is nearly parallel to the flow in order to reduce drag. ${ }^{2}$ For the simulations presented in the paper, a simple sinusoidal pattern is chosen for the angle of attack. A more complex calculation of the angle of attack is found in Ref. 24. The wingstrokes for the traditional dragonfly simulation are the following,

$$
\alpha_{F}(t)=45 \sin \left(2 \pi f_{F} t+\frac{\pi}{2}\right) \quad \text { and } \quad \zeta_{H}(t)=45 \sin \left(2 \pi f_{H} t+\frac{3 \pi}{2}\right),
$$

where the maximum angle of attack is 45 degrees. The forewings are mounted $6 \mathrm{~mm}$ forward of the aircraft center of mass at a distance along $\hat{b}_{y}$ axis equal to the constant radius of the cylinder outlined in Section A. The hindwings are mounted $9 \mathrm{~mm}$ rear of the aircraft center of mass. For initial conditions of $Z=5 \mathrm{~m}$ and $\theta=29^{\circ}$, the FWMAV climbs after five flapping cycles in Figure 7. However, without any control, the

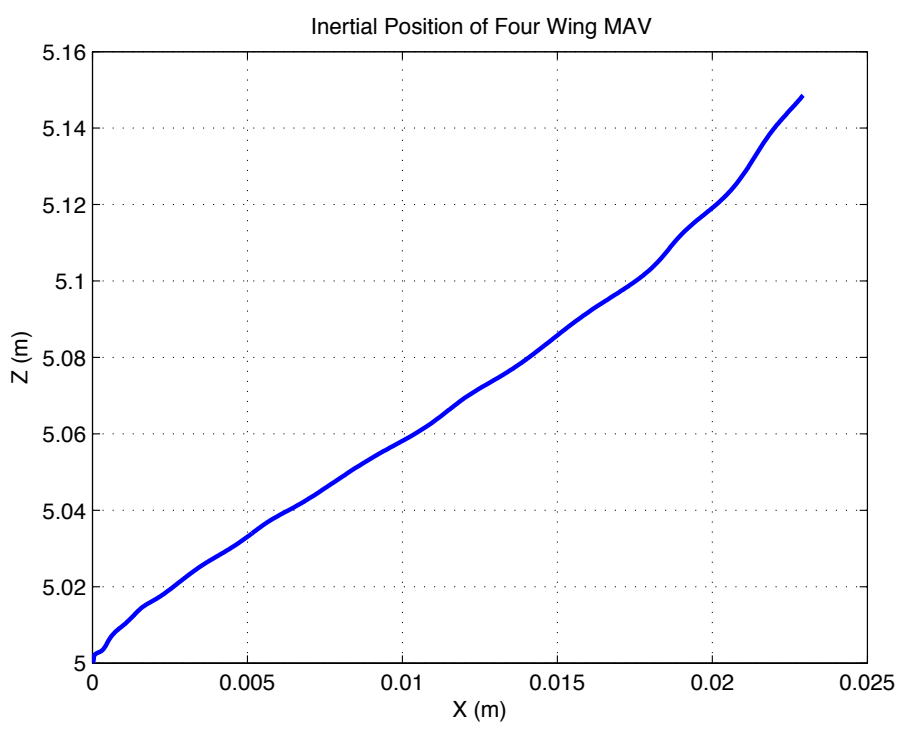

Figure 7. Dragonfly Simulation - Inertial Position

longitudinal mode is unstable and the pitching continues to increase in Figure 8. Eventually, without control, the central body of the FWMAV will 'noise over.'

The increase in the pitch angle is due to the aerodynamic pitching moment, as a result of the motion of the wings. The pitching velocity of the central body, $q$, is shown in Figure 9. The initial motion of the forewings is forward (downstroke), towards the nose of the aircraft. The initial gravity moment of the wings causes the negative pitch angle. However, the aerodynamic pitching moment takes over and causes the instability in the pitch axis of the FWMAV. The small yaw and roll angles are due to the asymmetry in the size of the wings. The hindwings have more mass and inertia than the forewings, resulting in the lack of 'perfect' cancellation of the rolling and yawing moments. If the forewings and hindwings have the same mass and size, the yaw and rolling motion is not present.

\section{Revised Dragonfly}

The wing placement differs from the normal dragonfly in that the wing placement is symmetrical about the central body center of mass. Furthermore, the wingstroke is altered to eliminate the effect of the gravity moment of the wings on the pitch angle of the central body. The stroke plane inclination is reduced to zero and the wingstrokes are designed to be equal and opposite for the forewings and hindwings. The forewings are placed at $7 \mathrm{~mm}$ forward of the central body and the hindwings at $7 \mathrm{~mm}$ aft of the origin of the $B$ frame. The forewings and hindwings have identical mass, chord, and span parameters. The wingstrokes for the forewings and hindwings are governed by

$$
\zeta_{F}(t)=20.25+20.25 \sin \left(2 \pi f t+\frac{3 \pi}{2}\right) \quad \text { and } \quad \zeta_{H}(t)=-20.25-20.25 \sin \left(2 \pi f t+\frac{3 \pi}{2}\right),
$$



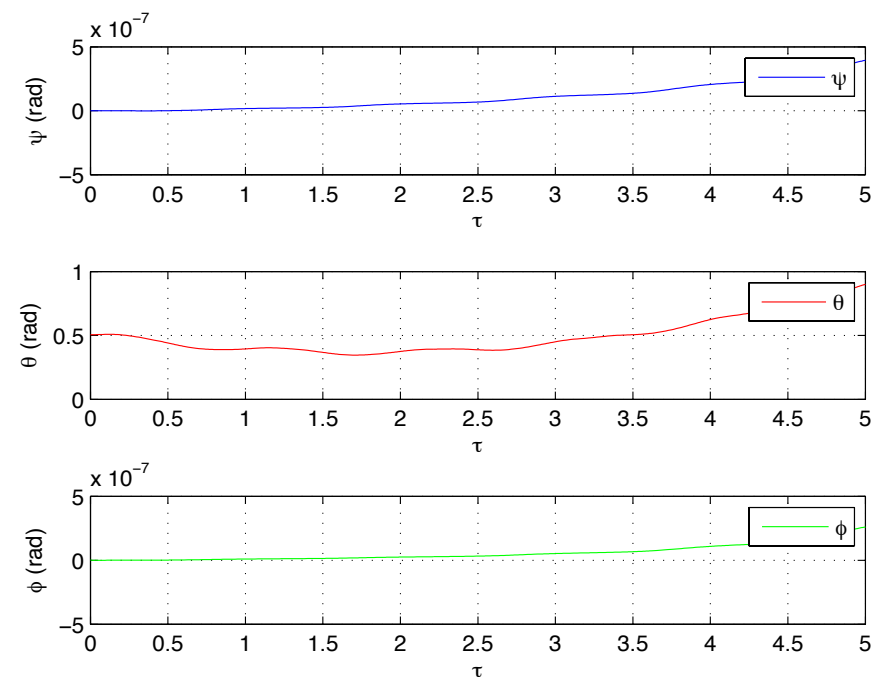

Figure 8. Traditional Dragonfly - Euler angles
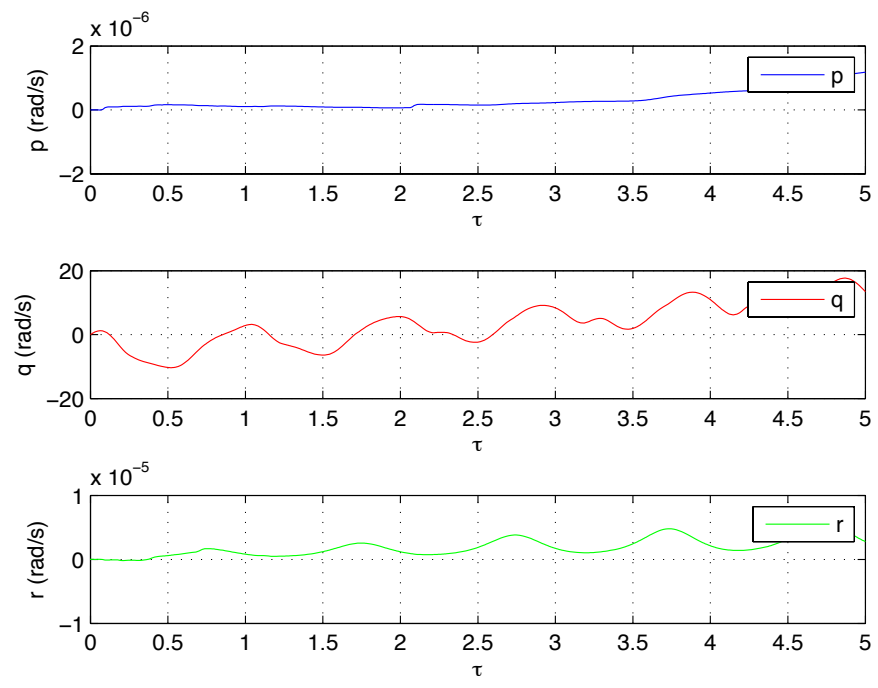

Figure 9. Traditional Dragonfly - Angular Velocity

where the phase shift is set to ensure an initial angle of zero and maximum amplitude of 40.5 degrees. The angle of attack for all of the wings are the following

$$
\alpha_{F}(t)=45 \sin (2 \pi f t) \quad \text { and } \quad \alpha_{H}(t)=45 \sin (2 \pi f t+\pi)
$$

The flapping frequency is equal for all four wings and is set to $39 \mathrm{~Hz}$. For an initial height of $Z=5 \mathrm{~m}$ and orientation parallel to the inertial frame, the inertial position of the altered dragonfly is shown in Figure 10. Due to perfect cancellation of aerodynamic moments, the orientation of the body remains parallel to the inertial frame. The flapping amplitude of the wings is designed to maintain a nearly consistent vertical position. The revised configuration does help the open loop stability of the pitch angle of the aircraft, in Figure 11. The angular velocity output, in Figure 12, shows nearly zero angular rates for the central body. The revised configuration, based on the change in position of the wing joints and altered wing strokes, shows excellent open loop stability in both attitude and position. One problem with the results presented is the 


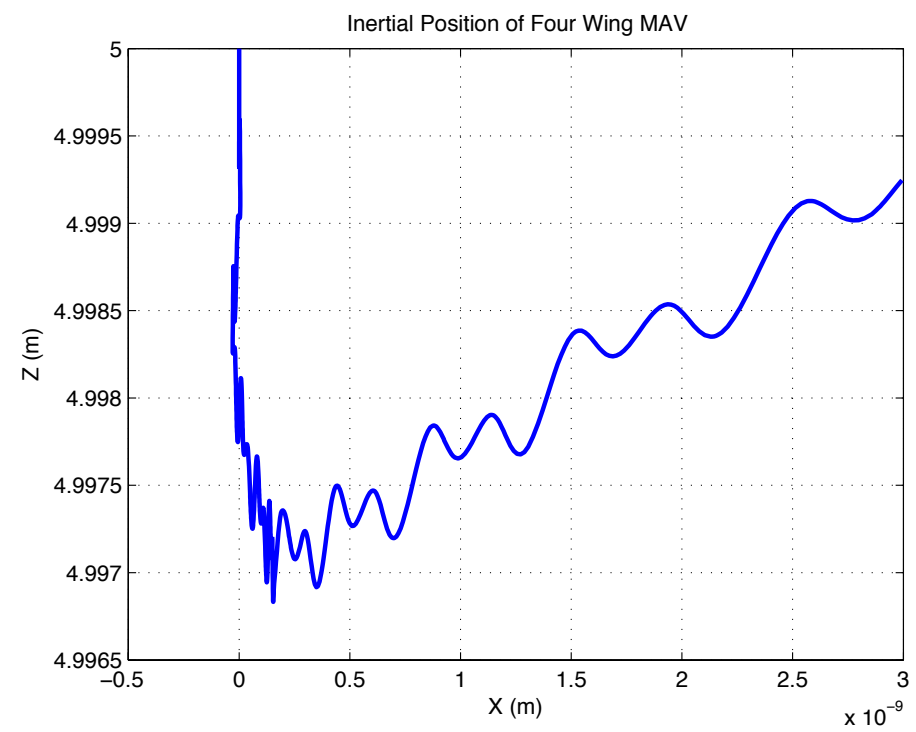

Figure 10. Revised Dragonfly Simulation - Inertial Position
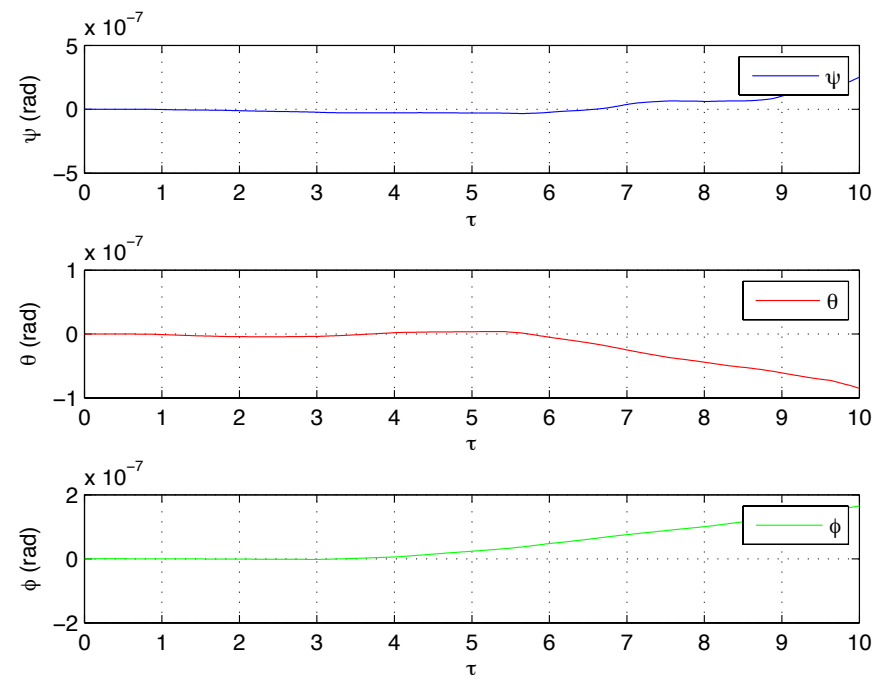

Figure 11. Revised Dragonfly - Euler angles

stroke amplitude required to maintain the initial position. The total stroke amplitude is $41^{\circ}$; at the end of the strokes the wings will nearly reach the noise and tail of the aircraft. A different wing shape, or faster flapping frequency, may reduce the requirement for the stroke amplitude. However, the wing parameters were chosen based on a biomimetic dragonfly wing. Also, the assumption of zero aerodynamic interaction between the wings will need to be investigated when the wing strokes are at their closest approach (when $\zeta \equiv 0)$.

\section{X-Wing 1}

The wings are arranged similar to the inspiration presented in Ref. 8 . The stroke plane is horizontal, $\beta \equiv 0$. Two cases are presented: the top wings leading the bottom wings by 180 degrees and the wings beating in-phase. The top wings are equivalent to the forewings in the dragonfly simulations and the bottom wings 

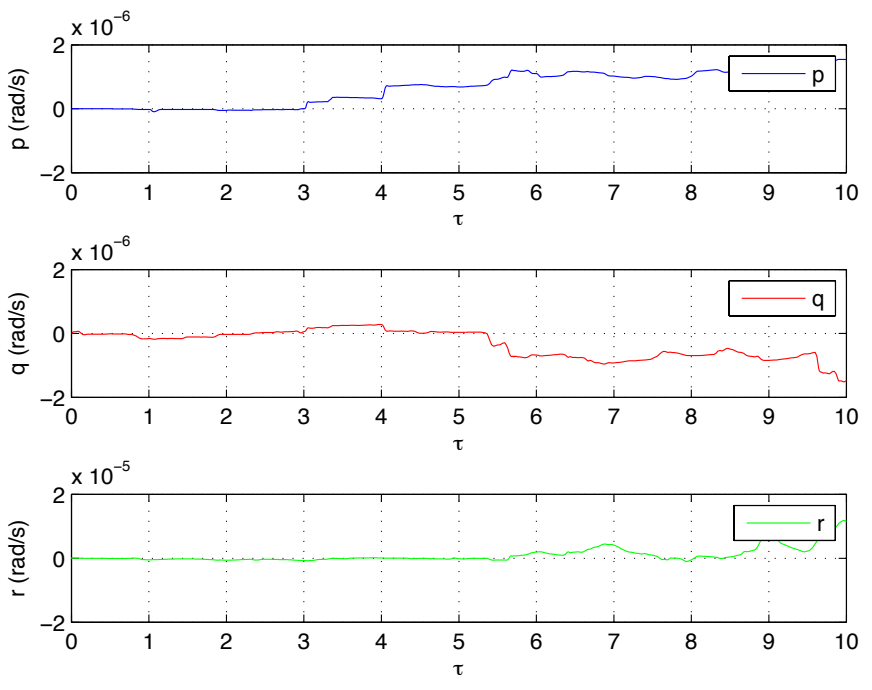

Figure 12. Revised Dragonfly - Angular Velocity

are equivalent to the hindwings, in regards to the derivation of the equations of motion. The wing parameters (span, chord, mass) for all four wings are identical and are set to equal the parameters of the forewings in the traditional dragonfly simulations. All four wings flap at a frequency of $39 \mathrm{~Hz}$. The wing kinematics for the wings, when the top wings are leading the bottom wings, are

$$
\zeta_{F}(t)=30 \sin (2 \pi f t+\pi) \quad \text { and } \quad \zeta_{H}(t)=30 \sin (2 \pi f t)
$$

and

$$
\alpha_{F}(t)=45 \sin \left(2 \pi f t+\frac{3 \pi}{2}\right) \quad \text { and } \quad \alpha_{H}(t)=45 \sin \left(2 \pi f t+\frac{\pi}{2}\right) .
$$

When the wings are in-phase, the resulting kinematics, expressed in degrees, are

$$
\begin{gathered}
\zeta_{F}(t)=30 \sin (2 \pi f t) \quad, \quad \zeta_{H}(t)=30 \sin (2 \pi f t), \\
\alpha_{F}(t)=45 \sin \left(2 \pi f t+\frac{\pi}{2}\right) \quad \text { and } \quad \alpha_{H}(t)=45 \sin \left(2 \pi f t+\frac{\pi}{2}\right) .
\end{gathered}
$$

The wings are placed at angles of 45 degrees from the $\hat{b}_{y}$ and $\hat{b}_{z}$ axes. The vehicle starts an initial height of $5 m$ in both cases, with initial orientation parallel to the inertial frame.

In Figures 13 and 15, the $\mathrm{x}$-wing configuration produced enough lift to increase the altitude of the FWMAV. When the top wings lead, the FWMAV has a forward acceleration component. When the wings are in-phase, the FWMAV accelerates in the negative $Y$ direction. The configuration, with the wings inphase, shows a more stable orientation than with the wings out of phase. The top wings leading in phase is a terrible choice as the vehicle quickly pitches upward. The wings beating in-phase shows a steadily decreasing pitch angle, which could possibly be controlled in a closed-loop setting.

In both cases, the vehicle is stable in yaw and roll, as the symmetrical motion of the wings (with respect to the longitudinal axis of the central body) cancels any yaw and roll moments produced by the wings. The vertical acceleration of the vehicles is nearly the same and both are unstable in pitch. However, when the wings beat out-of-phase, the pitch instability occurs after approximately 10 flapping cycles. When the wings beat in-phase, the pitch instability occurs within four flapping cycles, approximately 2.5 times faster. 


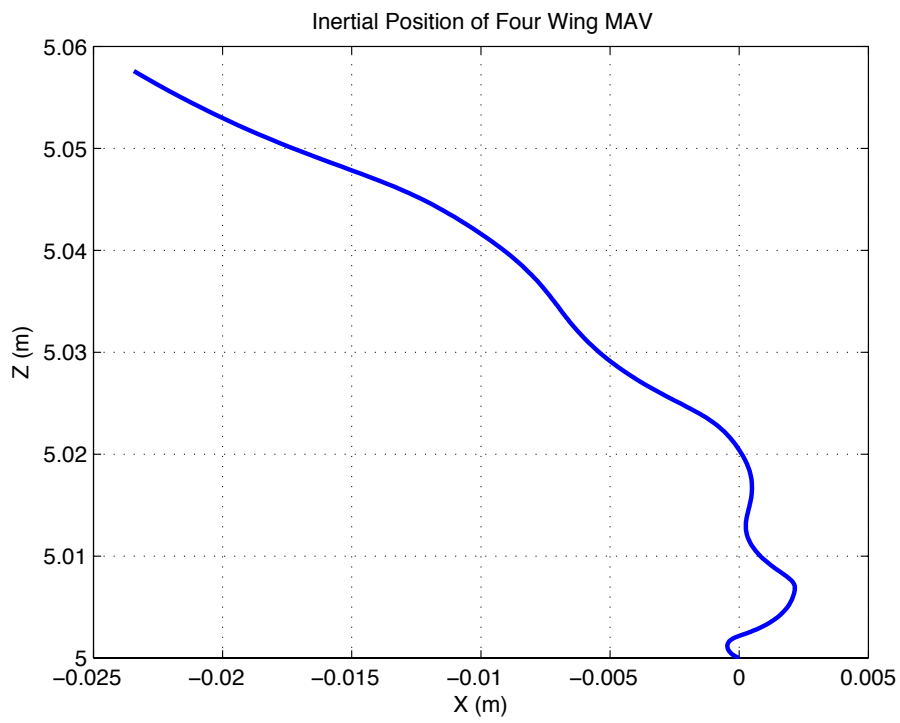

Figure 13. X-Wing1 - Wings In-Phase - Inertial Position
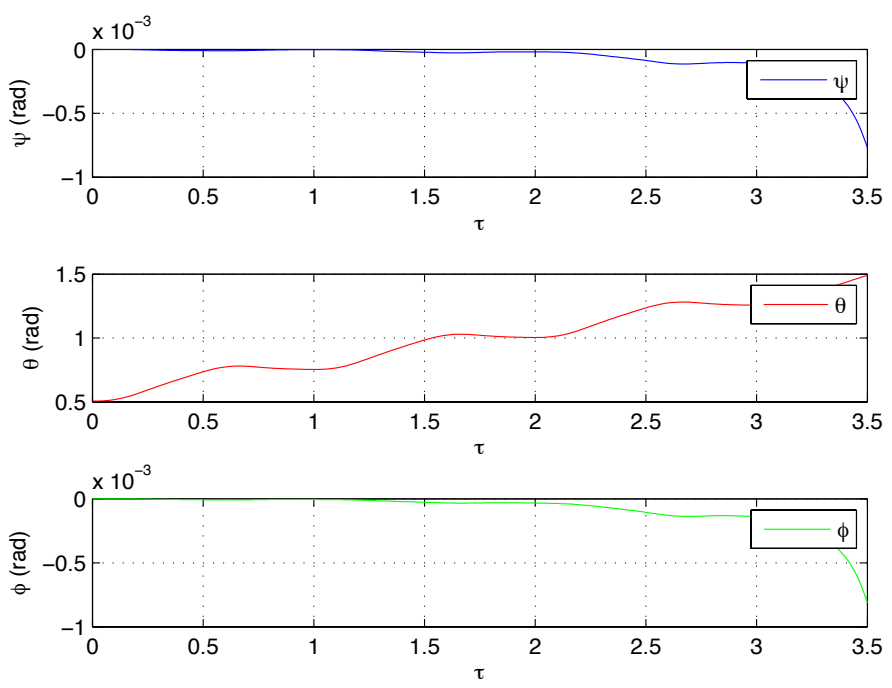

Figure 14. X-Wing1 - Wings In-Phase - Euler Angles

\section{E. X-Wing 2}

The wings are arranged as in Section D. The stroke plane is no longer identically zero, but equals 90 degrees. Two cases are presented: the wings flap opposite of each other and the wings flap in the same direction. When the wings flap opposite of each other, one wing goes 'up' while the other wing goes 'down', the wing stroke kinematics are the following

$$
\zeta_{F}(t)=20+20 \sin \left(2 \pi f t+\frac{\pi}{2}\right) \quad \text { and } \quad \zeta_{H}(t)=-20-20 \sin \left(2 \pi f t+\frac{3 \pi}{2}\right)
$$

and the angle of attack is governed by

$$
\alpha_{F}(t)=45 \sin (2 \pi f t+\pi) \quad \text { and } \quad \alpha_{H}(t)=45 \sin (2 \pi f t) .
$$




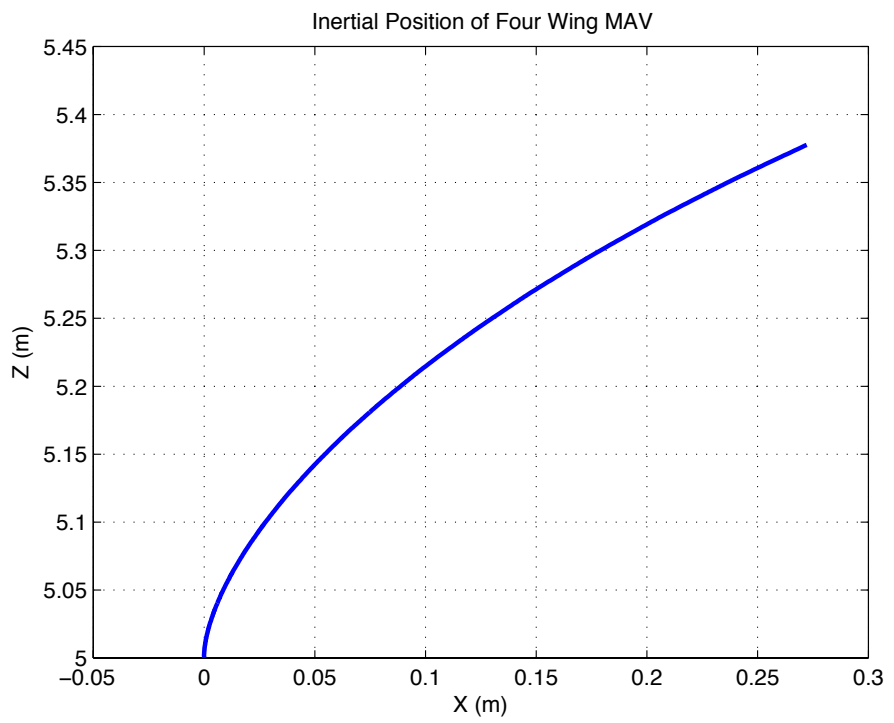

Figure 15. X-Wing1 - Wings Out-of-Phase - Inertial Position
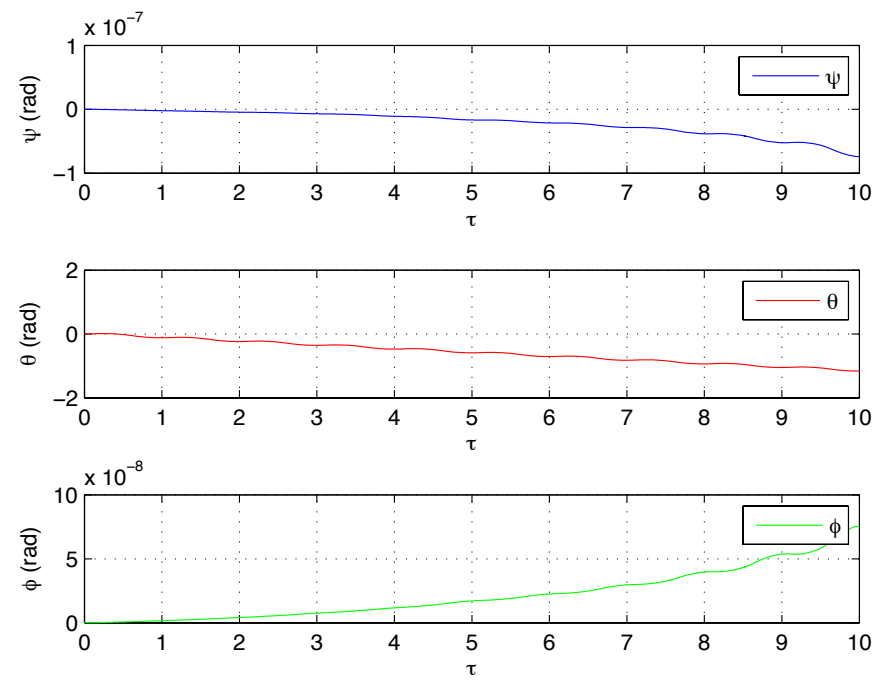

Figure 16. X-Wing1 - Wings Out-of-Phase - Euler Angles

When the wings flap in the same direction, both wings are going up and down at the same time, the wing stroke kinematics are

$$
\zeta_{F}(t)=20+20 \sin \left(2 \pi f t+\frac{3 \pi}{2}\right) \quad \text { and } \quad \zeta_{H}(t)=-20-20 \sin \left(2 \pi f t+\frac{3 \pi}{2}\right)
$$

and the angle of attack is governed by

$$
\alpha_{F}(t)=45 \sin (2 \pi f t) \quad \text { and } \quad \alpha_{H}(t)=45 \sin (2 \pi f t) .
$$

The inertial position of the FWMAV is presented in Figures 17 and 19. The orientation is presented in Figures 18 and 19. The given stroke parameters do not provide enough lift to keep the vehicle aloft, as the vehicle quickly descends. The simulations presented are with a flapping frequency of $100 \mathrm{~Hz}$. The speed of 
flapping, to keep the vehicle even remotely aloft, is a lot larger than the flapping frequency presented in the previous simulations. The wings and central body are the same size. The orientation of the vehicle is not necessarily an improvement over the normal dragonfly configurations and the previous x-wing simulations.

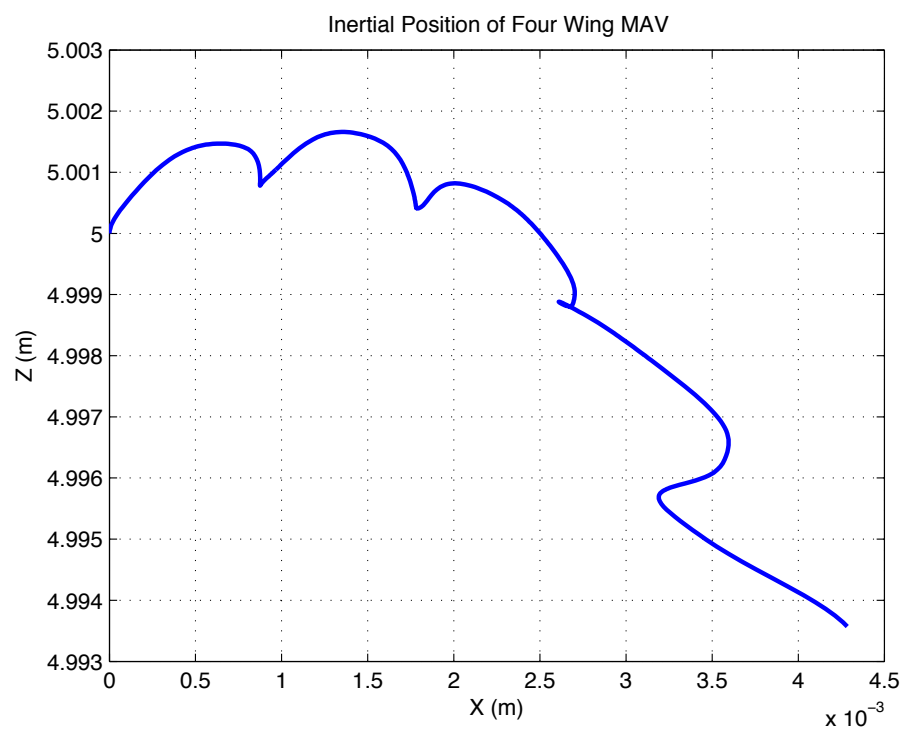

Figure 17. X-Wing2 - Same Direction - Inertial Position
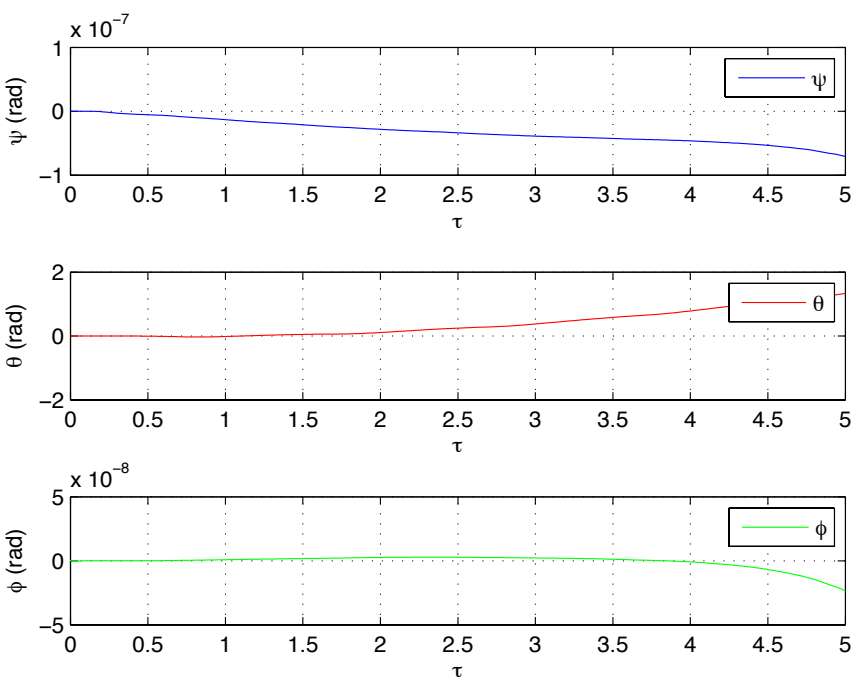

Figure 18. X-Wing2 - Same Direction - Euler Angles

The large flapping frequency may be a result of the aerodynamic model chosen for the simulations. However, since the configuration does not provide notably better stability than the other configurations presented, we conclude that the configuration is not a beneficial potential choice for vehicle design. 


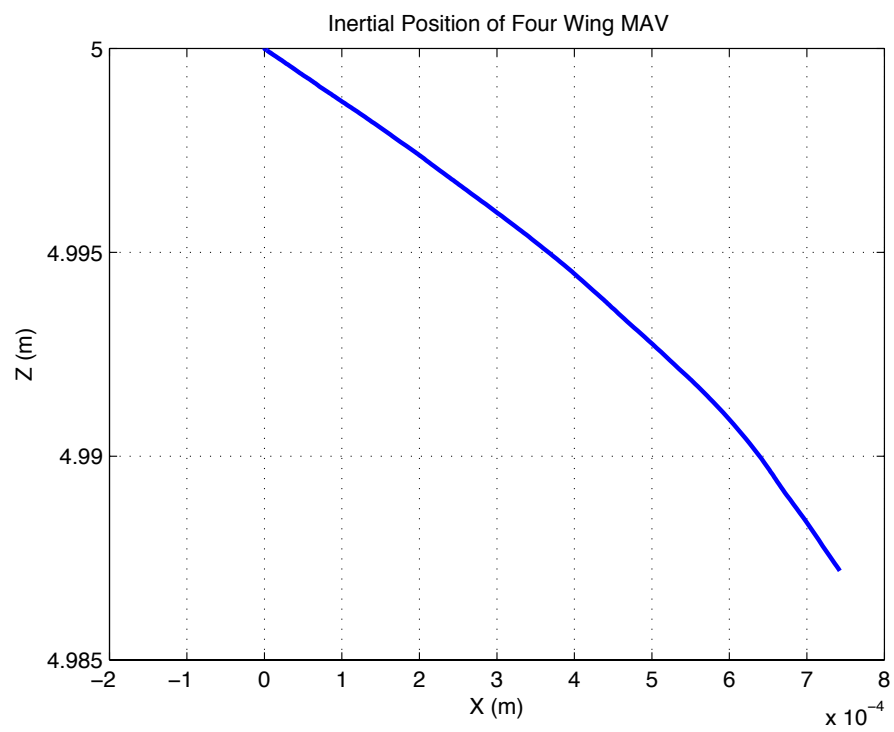

Figure 19. X-Wing2 - Opposite Direction - Inertial Position
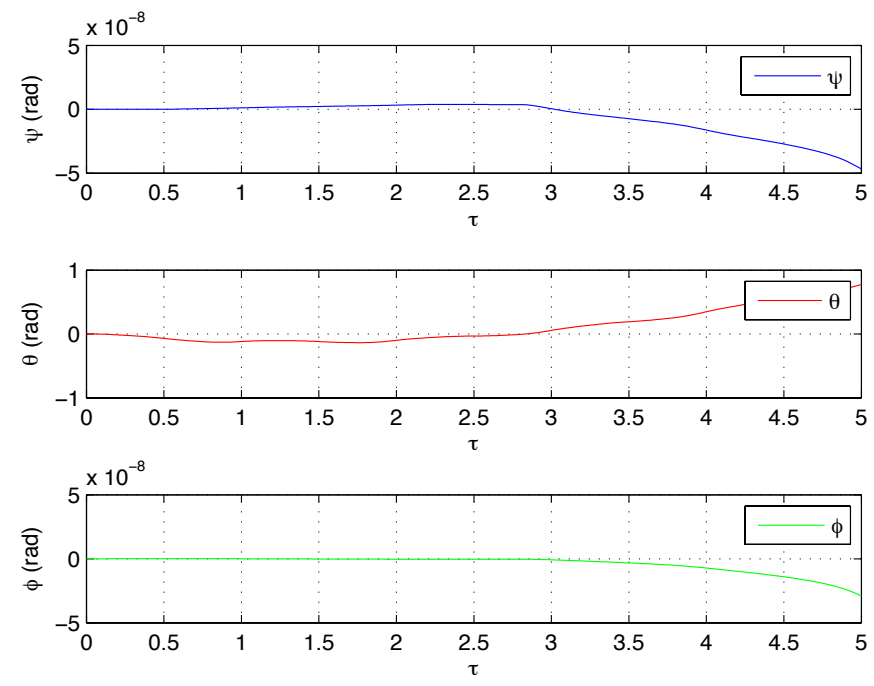

Figure 20. X-Wing2 - Opposite Direction - Euler Angles

\section{Conclusion}

The paper presents the derivation of a flight dynamics model for a four-wing, flapping wing micro-air vehicle. The dynamics model includes the inertial and mass effects of the wings on the central body and allows three degrees of freedom for each wing. Simulation results are presented to investigate the open loop stability, based on the dynamic model, of various four wing configurations and wing kinematics choices. The authors acknowledge that the possible design space for flapping wing micro-air vehicles is gigantic and that this study barely scratched the surface of the design space. From the initial simulations presented in this paper, the revised dragonfly configuration appear to have better open loop, inherent stability properties than a four wing vehicle modeled after a dragonfly. The $\mathrm{x}$-wing configuration, with the wingstrokes in a horizontal stroke plane, does not appear to provide any worse performance than the traditional dragonfly. The simulations presented do not include closed loop controls nor the effects of disturbances, e.g. wind 
gusts. Therefore, the dragonfly configuration is not ruled out due to the many wonderful aerodynamic feats of dragonflies and the demonstrated superior maneuverability and flight stability. Furthermore, if sufficient control authority exists at the wing joints, the revised dragonfly wingstrokes could be used for a hover condition and biomimetic wingstrokes could be used for turning and forward flight conditions. Future work will focus on varying configurations and wing kinematics to determine, if possible, better open loop designs for all of the configurations presented in this work.

\title{
References
}

\author{
${ }^{1}$ Wakeling, J. and Ellington, C., "Dragonfly Flight I. Gliding Flight and Steady-State Aerodynamic Forces," The Journal \\ of Experimental Biology, Vol. 200, 1997, pp. 543-556. \\ ${ }^{2}$ Norberg, R., "Hovering flight of the dragonfly Aeschna juncea L., kinematics and aerodynamics," Proceedings of the \\ Symposium on Swimming and Flying in Nature, California Institute of Technology, Pasadena, CA, July 8-12, 1974, Vol. 2, \\ 1975.
}

${ }^{3}$ Wakeling, J. and Ellington, C., "Dragonfly Flight II. Velocities, Accelerations, and Kinematics of Flapping Flight," The Journal of Experimental Biology, Vol. 200, 1997, pp. 557-582.

${ }^{4}$ Wakeling, J. and Ellington, C., "Dragonfly Flight III. Lift and Power Requirements," The Journal of Experimental Biology, Vol. 200, 1997, pp. 583-600.

${ }^{5}$ Wang, H., Zeng, L., Liu, H., and Yin, C., "Measuring wing kinematics, flight trajectory and body attitude during forward flight and turning maneuvers in dragonflies," The Journal of Experimental Biology, Vol. 206, 2003, pp. 745-757.

${ }^{6}$ DiLeo, C. and Deng, X., "Design and Experiments of a Dragonfly-Inspired Robot," 2008.

${ }^{7}$ Wakeling, J., "Odonatan Wing and Body Morphologies," Odonatologica, Vol. 26, 1997, pp. 35-52.

${ }^{8}$ Bolsman, C., Goosen, J., and F.Keulen, "Design Overview of a Resonant Wing Actuation Mechanism for Application in Flapping Wing MAVs," Proceedings of the 2009 European Micro-air Vehicle Conference and Competition 2009, Delft, Netherlands, 14-17 September 2009, 2009.

${ }^{9}$ Orlowski, C., Girard, A., and Shyy, W., "Derivation and Simulation of the Nonlinear Dynamics of a Flapping Wing Micro-air Vehicle," Proceedings of the 2009 European Micro-air Vehicle Conference and Competition 2009, Delft, Netherlands, 14-17 September 2009, 2009.

${ }^{10}$ Orlowski, C., Girard, A., and Shyy, W., "Pitch Control of a Flapping Wing Micro-air Vehicle Using a Tail and Control Mass," Accepted for the 2010 American Control Conference, Baltimore, Maryland, USA, 30 June - 02 July 2010, IEEE, 2010.

${ }^{11}$ Greenwood, D. T., Principles of dynamics, Prentice Hall, Upper Saddle River, NJ, 1988.

${ }^{12}$ Greenwood, D. T., Advanced dynamics, Cambridge University Press, 2006.

${ }^{13}$ Baruh, H., Analytical Dynamics, WCB McGraw-Hill, Boston, MA, 2006.

${ }^{14}$ Dickson, W., A.Straw, Poelma, C., and M.Dickinson, "An Integrative Model of Insect Flight Control," Proceedings of the 44th AIAA Aerospace Sciences Meeting and Exhibit, Reno, Nevada, USA, 9-12 January 2006, AIAA, 2006.

${ }^{15}$ Etkin, B. and Reid, L., Dynamics of flight, John Wiley and Sons, New York, NY, 1996.

${ }^{16}$ Khan, Z. and Agrawal, S., "Modeling and Simulation of Flapping Wing Micro Air Vehicles," Proceedings of IDETC/CIE'2005 2005 ASME International Design Engineering Technical Conferences, Long Beach, California, USA, September 24-28, 2005, ASME, 2005.

${ }^{17}$ Schenato, L., Analysis and Control of Flapping Flight: from Biological to Robotic Insects, Ph.D. thesis, University of California at Berkeley, California, Fall 2003.

${ }^{18}$ Deng, X., Schenato, L., Wu, W., and Sastry, S., "Flapping Flight for Biomimetic Robot Insects: Part I - System Modeling," IEEE Transactions on Robotics, Vol. 22, No. 4, August 2006.

${ }^{19}$ Deng, X., Schenato, L., and Sastry, S., "Flapping Flight for Biomimetic Robot Insects: Part II - Flight Control Design," IEEE Transactions on Robotics, Vol. 22, No. 4, August 2006.

${ }^{20}$ Doman, D., Oppenheimer, M., and Sigthorsson, D., "Dynamics and Control of a Minimally Actuated Biomimetic Vehicle: Part I - Aerodynamic Model," Proceedings of the AIAA Guidance, Navigation, and Control Conference, Chicago, Illinois, USA, August 10-13, 2009, AIAA, 2009.

${ }^{21}$ Oppenheimer, M., Doman, D., and Sigthorsson, D., "Dynamics and Control of a Minimally Actuated Biomimetic Vehicle: Part II - Control," Proceedings of the AIAA Guidance, Navigation, and Control Conference, Chicago, Illinois, USA, August 10-13, 2009, AIAA, 2009.

${ }^{22}$ Oppenheimer, M., Doman, D., and Sigthorsson, D., "Dynamics and Control of a Biomimetic Vehicle Using Biased Wingbeat Forcing Functions: Part I - Aerodynamic Model," Proceedings of the 48th AIAA Aerospace Sciences Meeting Including the New Horizons Forum and Exposition, Orlando, Florida, USA, January 4-7, 2010, AIAA, 2010.

${ }^{23}$ Doman, D. and Sigthorsson, M. O. D., "Dynamics and Control of a Biomimetic Vehicle Using Biased Wingbeat Forcing Functions: Part II - Control," Proceedings of the 48th AIAA Aerospace Sciences Meeting Including the New Horizons Forum and Exposition, Orlando, Florida, USA, January 4-7, 2010, AIAA, 2010.

${ }^{24}$ Sun, M. and Lan, S., "A computational study of the aerodynamic forces and power requirements of dragonfly (Aeschna juncea) hovering," The Journal of Experimental Biology, Vol. 207, 2004, pp. 1887-1901. 